Eine mögliche Erklärung bietet ein anderer Effekt des Nebenwahlcharakters von europäischen Wahlen: In Antizipation des tendenziell höheren Stimmenverlustes von Regierungsparteien könnte die CDU hier bewusst ein besonderes Augenmerk auf dieses in der Öffentlichkeit bedeutende Thema gelegt haben.

Ebenfalls erklärungsbedürftig ist die Position der SPD. Ihre Wählerschaft ist über die Geheimdienstaffäre überdurchschnittlich stark verunsichert, aber das Wahlprogramm spiegelt dies vor allem auch qualitativ gesehen nicht wider. Die Partei entspricht mit ihren Positionen zur Inneren Sicherheit bereits früher nicht dem programmatischen Willen ihrer sicherheitssensiblen Wähler. Hier sind zwei Gründe vorstellbar: Die SPD ist sich über die sicherheitspolitischen Präferenzen ihrer Wählerschaft nicht im Klaren, oder die sicherheitspolitische Position der SPD-nahen Wähler spielt insgesamt eine untergeordnete Rolle bei der Wahlentscheidung, so dass die Partei diesem Punkt bei der Ausarbeitung des Manifests aus rationalen Kostenerwägungen weniger Bedeutung beimisst. Der Befund gilt gleichermaßen für die Labour Party. Interessant wäre daher ein systematischer Quervergleich sozialdemokratischer Parteien in Europa.

Offenkundig ist die Aufmerksamkeit für bestimmte Aspekte der Inneren Sicherheit bei den europäischen Wahlen nationalstaatlich geprägt: Innere Sicherheit spielt für die britischen Parteien insbesondere dort eine Rolle, wo sie die nationale Souveränität tangiert, nationale Interessen befördert und den britischen Bürgern dient. Die deutschen Parteien beziehen etwas stärker die europäische Innere Sicherheit und erst in zweiter Linie die nationale mit in die Programmatik ein, wobei es im nationalen Diskurs dann aber stärker um die Befugnisse von Sicherheitsbehörden und die Einschränkung individueller Freiheitsrechte geht.

\title{
Zum Sozialprofil der deutschen Abgeordneten nach der Wahl zum Europäischen Parlament 2014
}

\section{Peter Rütters}

Wie bei jeder vorangegangenen Wahl zum Europäischen Parlament (EP) gab es auch bei der 8. Direktwahl im Mai 2014 einige Rahmenbedingungen, die in besonderer Weise Einfluss hatten auf die Wahlchancen der beteiligten Parteien, auf die Nominierung von Kandidaten und deren Möglichkeit, ein Mandat zu erlangen. Aufgrund der Deckelung der Gesamtzahl der Mitglieder des Europäischen Parlaments (MdEP) verminderte sich die Anzahl der in Deutschland zu wählenden Abgeordneten geringfügig von 99 auf 96. ${ }^{1}$ Auf die Vertretungschancen der Parteien im EP wirkte sich diese Veränderung aber kaum aus. Von größerer Bedeutung für den Wahlausgang und die im EP vertretenen Parlamentarier waren die Urteile des Bundesverfassungsgerichts, Sperrklauseln von fünf und drei Prozent für die

1 Gemäß Art. 14, Abs. 2 des EU-Vertrags wurde die Anzahl der Abgeordneten des EP auf 750 (zuzüglich des Präsidenten) festgelegt und die Höchstzahl der Sitze, die ein Mitgliedstaat erhalten kann, auf 96. 
EP-Wahlen in Deutschland für verfassungswidrig zu erklären. ${ }^{2}$ Die daraus folgende Veränderung des Wahlverfahrens konnte sich auf die Nominierungspraxis ${ }^{3}$ der Parteien auswirken und das Abstimmungsverhalten der Wähler beeinflussen. Auf jeden Fall hatte sie Folgen für die Anzahl der im EP vertretenen Parteien und die Zuweisung von Mandaten.

Zwar liegt die Annahme nahe, dass der Wegfall der Sperrklausel Wähler dazu animieren würde, für Parteien zu stimmen, deren Einzug ins Europäische Parlament bisher an der Fünf-Prozent-Hürde gescheitert war. Doch weisen die Stimmenzahlen und die Stimmenanteile für die Parteien, die auch bei der Wahl von 2014 weit unterhalb der Fünf-ProzentHürde blieben, aber aufgrund der veränderten Zugangsvoraussetzungen ein Mandat im EP erlangen konnten, diesen Effekt nicht oder nur in sehr geringem Umfang auf. Die Wahlergebnisse der etablierten Parteien wurden durch Wählerwanderungen zu diesen Parteien nicht sonderlich beeinflusst. ${ }^{4}$

Bis zur 7. Wahlperiode (2009 bis 2014) erreichten wegen der Zugangsbeschränkung durch die Sperrklausel höchsten sechs Parteien den Einzug ins EP. Das wäre auch 2014 nicht anders gewesen, da an die Stelle der FDP (Stimmenanteil: 3,4 Prozent) die Alternative für Deutschland (AfD) getreten wäre. Außer der AfD, die bei einem Stimmenanteil von 7,0 Prozent mit sieben Abgeordneten erstmals im EP vertreten ist, konnten durch den Wegfall der Sperrklausel sieben weitere Parteien mit jeweils nur einem Sitz ins EP einziehen. Durch diese Ein-Mandat-Parteien und die Bewahrung der EP-Repräsentanz für die

2 Vgl. die Urteile des Bundesverfassungsgerichts vom 9. November 2011 (2 BvC 4/10, 2 BvC 6/10, 2 BvC 8/10) und zuletzt vom 26. Februar 2014 (2 BvE 2/13 u.a., 2 BvR 2220/13 u.a.); danach seien Sperrklauseln für die in Deutschland durchzuführenden Wahlen zum EP „unter den gegenwärtigen rechtlichen und tatsächlichen Verhältnissen nicht zu rechtfertigen“".

3 Da aber der innerparteiliche Entscheidungsprozess über die Nominierung von Kandidaten und die Zusammenstellung der Kandidatenlisten von verschiedenen Faktoren beeinflusst wird, lässt sich das Nominierungsergebnis nicht auf einen zentralen Faktor zurückführen, vgl. hierzu Benjamin Höhne, Rekrutierung von Abgeordneten des Europäischen Parlaments. Organisation, Akteure und Entscheidungen in Parteien, Opladen 2013.

4 Bezogen auf die Fünf-Prozent-Hürde hat keine der sieben Parteien, die 2014 zum ersten Mal ein Mandat im EP erreichen konnten und weniger als fünf Prozent der Stimmen erhielten, einen markanten Stimmenzuwachs im Vergleich zur Wahl 2009 erfahren: Die Freien Wähler - die stimmenstärkste Kleinstpartei - hat mit 428.524 Stimmen (2014) gegenüber der Wahl 2009 etwa 14.000 Stimmen eingebüßt, während die Piraten mit 424.510 Stimmen (2014) fast 200.000 Stimmen hinzugewinnen konnten; die Tierschutz-Partei konnte sich mit 366.303 Stimmen (2014) um 76.609 Stimmen steigern; die Familien-Partei hat mit 202.871 Stimmen (2014) fast 50.000 Stimmen eingebüßt, während die ÖDP, die 185.119 Stimmen (2014) erhielt, sich um ungefähr 50.000 Stimmen verbessern konnte. Erfolgreich war Die Partei, sie erhielt bei ihrer ersten Kandidatur 184.525 Stimmen; schließlich votierten für die NPD, die 2009 nicht zur Wahl angetreten war, 300.815 Wähler, gleichzeitig büßte die REP fast 240.000 Stimmen ein und bekam 2014 nur noch 109.856 Stimmen, so dass sie den Einzug ins EP verpasste. Der saldierte Stimmengewinn dieser sieben Kleinstparteien liegt bei 2,5 Prozent der gültigen Stimmen (beziehungsweise 1,7 Prozent, wenn eine Wählerwanderung von den REP-Wählern zur NPD angenommen wird). Dennoch ist festzuhalten: Das Abstimmungsverhalten der Wahlberechtigten wurde durch den Wegfall der Sperrklausel nicht in relevantem Umfang beeinflusst. Gewinner ist die FDP, die trotz des Stimmenanteils von nur 3,4 Prozent weiter im EP vertreten ist. Verlierer ist das Parlament, das mit sieben beziehungsweise acht Parteien belastet ist, deren geringe Mitgliederzahl keine und auch über eine Fraktionsbindung nur eine sehr begrenzte konstruktive Parlamentspolitik erlaubt. Zu den Ergebnissen der EP-Wahl 2014 vgl. auch den Beitrag von Oskar Niedermayer in diesem Heft. 
FDP mit drei Abgeordneten verminderte sich die Anzahl der auf die etablierten Parteien zu verteilenden Parlamentssitze rechnerisch um zehn.

Zugunsten der Ein-Mandat-Parteien wirkte sich das seit 2009 angewandte Verfahren zur Verteilung der Parlamentssitze nach Sainte-Laguë/Schepers aus. ${ }^{5}$ Dieses Berechnungsverfahren löste die Hare/Niemeyer-Methode mit der Intention ab, die bisherige Benachteiligung kleiner Parteien zu vermeiden. Nicht in Betracht gezogen wurde die Auswirkung des Verteilungsverfahrens nach Sainte-Laguë/Schepers, wenn auf die regulierende Wirkung einer Sperrklausel verzichtet würde. Der Wegfall jeglicher Hürden führt tendenziell zu einer einflussmindernden Fragmentierung der parlamentarischen Repräsentation der Bürger.

Einfluss auf den Ausgang der Wahlen und die Chancen der bislang im EP vertretenen Parteien, ihre Position zu bewahren oder durch Stimmen- und Mandatsgewinne sogar zu verbessern, hatten Veränderungen des innerdeutschen und europapolitischen Umfeldes. Die kleineren Parteien - FDP, Die Grünen und Die Linke - vermochten bei den Europawahlen 2004 und 2009 durch bundespolitische Ereignisse, die die großen Parteien (vor allem die SPD) belasteten (unter anderem so genannte Hartz-Gesetze, die Große Koalition von 2005 bis 2009), bemerkenswerte Wahlerfolge zu verzeichnen, was den Charakter der EP-Wahlen als „Sekundärwahlen“ bestätigte. ${ }^{6} 2014$ war es ihnen nicht mehr möglich, solche elektoralen Windfall-Profite zu realisieren. ${ }^{7}$ Bis zur Europawahl war allerdings nicht abzuschätzen, welche Parteien von den erwarteten Stimmen- und Mandatseinbußen der kleinen Parteien profitieren könnten.

Angesichts der auf den ersten Blick beachtlichen Veränderungen für die im Europäischen Parlament vertretenen deutschen Parteien und Abgeordneten als Folge der Besonderheiten der Wahl 2014 liegt die Frage nahe, ob und welche Auswirkungen daraus für das Sozialprofil der Europa-Abgeordneten resultieren. Damit soll die in Heft 4/2013 der

5 Bei der Europa-Wahl 2014 hat die Anwendung des Sitzverteilungsverfahrens nach Sainte-Laguë/ Schepers eine beachtliche Verzerrung bei der Stimmenanforderung für ein Mandat zur Folge. Im Durchschnitt wären bei 29.340.700 gültigen Stimmen und 96 zu verteilenden Sitzen 305.632 Stimmen für ein Mandat erforderlich. Vier Parteien - Die Partei (184.525), ÖDP (185.119), Familien-Partei (202.871) und die NPD (300.815) - benötigten zum Teil erheblich weniger Stimmen für ein Mandat als diesen Durchschnittswert (zum Teil nur 60 Prozent), während andere Kleinstparteien - Freie Wähler (428.524), Piraten (424.510) und Tierschutzpartei (366.303) - mit Stimmenanteilen von bis zu 40 Prozent über diesem Durchschnittswert ebenfalls nur ein Mandat erhielten.

6 Vgl. Jürgen Mittag / Claudia Hülsgen, Von Sekundärwahlen zu europäischen Wahlen? 30 Jahre Direktwahlen zum Europäischen Parlament, in: integration, 32. Jg. (2009), H. 2, S. $105-122$, S. 107; zu den Europawahlen 2004 und 2009 vgl. Oskar Niedermayer, Die Wahl zum Europäischen Parlament vom 13. Juni 2004 in Deutschland: Ein schwarzer Tag für die SPD, in: ZParl, 36. Jg. (2005), H. 1, S. 3 - 19; ders., Die Wahl zum Europäischen Parlament vom 7. Juni 2009 in Deutschland: SPD-Debakel im Vorfeld der Bundestagswahl, in: ZParl, 40. Jg. (2009), H. 4, S. $711-731$.

7 Insbesondere die nach zehn Jahren ohne parlamentarische Präsenz bei den Wahlen 2004 und 2009 sehr erfolgreiche FDP trat 2014 mit einer mehrfachen Bürde an. Nach vielen Rückschlägen bei den Landtagswahlen seit 2009 zeigte sich die FDP, die ihr bürgerrechtlich-liberales politisches Profil zugunsten eines klientelistischen Agierens in der christlich-liberalen Koalition (2009 bis 2013) weitgehend aufgegeben hatte, bei den Bundestagswahlen 2013 nicht mehr in der Lage, eine ausreichende Anzahl von Wählern zu mobilisieren, um erneut in den Bundestag einziehen zu können. Hinzu kamen ernsthafte Personalprobleme (siehe Anmerkung 9). 
ZParl veröffentlichte Darstellung zur Sozialstruktur der deutschen EP-Abgeordneten ${ }^{8}$ aktualisiert werden.

\section{Kontinuität und Erneuerung bei der Nominierung für die Europa-Wahl 2014}

Auf den ersten Blick scheint die Nominierungspraxis der bisher im EP vertretenen Parteien nicht auffällig von den vorangegangenen Wahlperioden abzuweichen. Alle Parteien zusammengefasst lag die Kontinuitätsquote bei der Re-Nominierung bisheriger MdEP bei 71,7 Prozent (bezogen auf die 99 Mandate der 7. Wahlperiode), was in etwa dem Durchschnitt der Erneuerungsquote früherer Wahlperioden von etwa 30 Prozent entspricht. Die Nominierungspraxis für die EP-Wahl 2014 kennzeichnete dennoch eine größere personelle Kontinuität, als dies in Tabelle 1 zum Ausdruck kommt.

Betrachtet man die Nominierungspraxis der FDP im Detail, erweist sich die „Erneuerungsquote" von 41,7 Prozent (gemäß den oben genannten Bedingungen) bei der Kandidatenaufstellung als Folge parteiinterner Personalprobleme. Nach der Aufstellung von sieben bisherigen Mitgliedern des EP standen - mit Ausnahme des ambitionierten Holger Krahmer - keine weiteren FDP-Abgeordneten der auslaufenden Wahlperiode zur Verfügung. ${ }^{9}$ Bei den Grünen wurden neun von $14 \mathrm{MdEP}$ erneut nominiert; zumindest zwei der fünf nicht wieder aufgestellten hatten die Absicht gehabt, nochmals zu kandidieren. ${ }^{10}$ Die

8 Vgl. Peter Rütters, Daten zur Sozialstruktur der deutschen Abgeordneten des Europäischen Parlaments, in: ZParl, 44. Jg. (2013), H. 4, S. 759 - 782; ders., „Verbleib“ von in Deutschland gewählten Europa-Abgeordneten, in: ZParl, 44. Jg. (2013), H. 4, S. 783 - 803. In beiden Aufsätzen finden sich Hinweise auf die einschlägige Sekundärliteratur. Um Redundanzen so gering wie möglich zu halten, sollen diese hier nicht wiederholt werden. Ergänzend sei auf zwei Aufsätze zum Thema aufmerksam gemacht: Jürgen Mittag / Diana Wendland, Zwischen Individuum und Typus: Die deutschen sozialdemokratischen Abgeordneten des Europäischen Parlaments (19522011) in kollektivbiografischer Perspektive, in: Mitteilungsblatt des Instituts für soziale Bewegungen, Bd. 45 (2011), S. 205 - 245; Jürgen Mittag, Europaabgeordnete zwischen transnationaler Repräsentation und politischem Abstellgleis. Zuschreibungen, Selbstverständnis und Biografien der MdEP im Lichte historiografischer Forschungsperspektiven, in: Historische Mitteilungen, Bd. 23 (2010), S. $64-86$.

9 Von den zwölf FDP-Abgeordneten der siebten Wahlperiode konnten drei Spitzenkandidaten (Alexander Pickart Alvaro, Georgios Chatzimarkakis, Silvana Koch-Mehrin) nicht erneut nominiert werden beziehungsweise verzichteten auf eine erneute Kandidatur; zwei mussten die Aberkennung ihres Doktortitels wegen nachgewiesener Plagiate hinnehmen (Chatzimarkakis und KochMehrin), einer (Pickart Avaro) muss sich gerichtlich als Verursacher eines Autounfall unter Drogeneinfluss wegen fahrlässiger Tötung verantworten. Ein Abgeordneter, Jürgen Creutzmann (geboren 1945), zum Zeitpunkt der Europawahl fast 70 Jahre alt, verzichtete aus Altersgründen auf eine erneute Kandidatur. Einzig Holger Krahmer, der insbesondere als wenig sachkompetenter „Klimaskeptiker" von sich reden gemacht hatte, scheiterte in einer Kampfabstimmung um die Nominierung für den fünften Listenplatz der FDP für die EP-Wahl.

10 Gerald Häfner und Elisabeth Schröter erhielten nicht mehr die notwendige Unterstützung auf dem Dresdner Nominierungsparteitag (7. bis 9. Februar 2014), um auf einen akzeptablen Listenplatz zu gelangen. Andere - Werner Schulz (geboren 1950) und Heide Rühle (geboren 1948) - verzichteten auf eine erneute Kandidatur, vermutlich aus Altersgründen. Hiltrud Breyer, die im Oktober 2013 als Nachrückerin für Franziska Brantner nochmals in das EP gelangte, aber bereits bei der Listenaufstellung 2009 umstritten war und nur auf einem aussichtslosen Listenplatz (Platz 15) rangierte, erreichte den erneuten Einzug ins Europäische Parlament zu spät, um noch Chancen haben zu können, bei der Kandidatenaufstellung für die Wahl 2014 berücksichtigt zu werden. 


\begin{tabular}{|c|c|c|c|c|c|c|c|}
\hline Parteien & $\begin{array}{l}\text { MdEP } \\
2009 \text { bis } \\
2014\end{array}$ & $\begin{array}{l}\text { davon: } \\
2014 \\
\text { erneut } \\
\text { nominiert }\end{array}$ & $\begin{array}{l}\text { davon: } \\
2014 \\
\text { erstmals } \\
\text { nominiert }\end{array}$ & $\begin{array}{c}\text { Kandidaten } \\
\text { mit diskonti- } \\
\text { nuierlicher } \\
\text { EP-Mitglied- } \\
\text { schaft } \\
\end{array}$ & $\begin{array}{l}\text { Konti- } \\
\text { nuität } \\
\text { (in } \\
\text { Prozent) }\end{array}$ & $\begin{array}{l}\text { EP- } \\
\text { Mandate } \\
2014\end{array}$ & $\begin{array}{c}\text { Kontinui- } \\
\text { tätsquote der } \\
\text { realisierten } \\
\text { Mandate }\end{array}$ \\
\hline CDU & 34 & 26 & 8 & - & 76,4 & 29 & $86,2^{\mathrm{e}}$ \\
\hline CSU & 8 & 7 & 1 & - & 87,5 & 5 & 100,0 \\
\hline SPD & 23 & $16^{\mathrm{b}}$ & 6 & $1^{\mathrm{c}}$ & $69,5^{\mathrm{d}}$ & 27 & $63,0^{f}$ \\
\hline Die Grünen & 14 & 9 & 5 & - & 64,2 & 11 & 81,8 \\
\hline FDP & 12 & 7 & 5 & - & 58,3 & 3 & 100,0 \\
\hline Die Linke & 8 & 6 & 2 & - & 75,0 & 7 & 85,7 \\
\hline insgesamt & 99 & 71 & 27 & 1 & 71,7 & 82 & 79,3 \\
\hline \multirow{4}{*}{\multicolumn{8}{|c|}{$\begin{array}{l}\text { Bezugsgröße für „erneut nominierte“ (Spalte 3) und „,erstmals nominierte“ (Spalte 4) Kandidaten so- } \\
\text { wie die „Kontinuitätsquote“ (Spalte 6) ist die Anzahl der MdEP in der beziehungsweise am Ende der } \\
\text { 7. WP (insgesamt } 99 \text { Abgeordnete). Die „Kontinuitätsquote der realisierten Mandate“ gibt den Anteil } \\
\text { der Abgeordneten, die bereits in der 7. WP Mitglied des EP waren, an den MdEP der 8. WP (insge- } \\
\text { samt 82) an. } \\
\text { b } \\
\text { Hier nicht berücksichtigt der EP-Abgeordnete Norbert Neuser, der auf dem für die Nominierung aus- } \\
\text { sichtslos scheinenden Listenplatz } 27 \text { aufgestellt wurde. } \\
\text { c Sylvia-Yvonne Kaufmann war von } 1999 \text { bis } 2009 \text { MdEP für die PDS/Die Linke. Sie verließ die Partei } \\
2009 \text { und wechselte zur SPD, nachdem ihr wegen ihrer die EU befürwortenden Politik im Europä- } \\
\text { ischen Parlament nur noch ein aussichtsloser Platz auf der Liste der Linkspartei für die EP-Wahl } 2009\end{array}$}} \\
\hline & & & & & & & \\
\hline & & & & & & & \\
\hline & & & & & & & \\
\hline \multicolumn{4}{|c|}{$\begin{array}{l}\text { eingeräumt worden war. } \\
\text { d Ohne Sylvia-Yvonne Kaufmann und Norbert Neuser. }\end{array}$} & & & & \\
\hline \multirow{2}{*}{\multicolumn{8}{|c|}{$\begin{array}{l}\text { e Wegen der Aufstellung von Landeslisten wurden nur } 25 \text { von } 26 \text { re-nominierten MdEP der CDU } \\
\text { wiedergewählt. } \\
\text { f Insgesamt wurden } 17 \text { re-nominierte SPD-Kandidaten berücksichtigt, einschließlich Norbert Neuser } \\
\text { (siehe unter b). }\end{array}$}} \\
\hline & & & & & & & \\
\hline \multicolumn{8}{|c|}{ Quelle: Eigene Zusammenstellung. } \\
\hline
\end{tabular}

Linke zeigt im Vergleich zum Auswechseln fast aller EP-Abgeordneten bei der Kandidatenaufstellung 2009 (wegen europapolitischer Differenzen zwischen den Abgeordneten und der Parteispitze) bei der Nominierung für die 8. Direktwahl eine bemerkenswerte Kandidaten-Kontinuität: Sechs von acht MdEP wurden erneut nominiert. ${ }^{11}$ Von $23 \mathrm{MdEP}$ der SPD konnten 17 ihre Kandidatur erneuern (davon einer - Norbert Neuser - auf dem aussichtslos scheinenden Listenplatz 27 nominiert), während sechs nicht mehr aufgestellt wurden. Den sechs nicht erneut nominierten Abgeordneten war gemeinsam, dass sie zwischen 1946 und 1952 geboren wurden und mit dem Ende der 7. Wahlperiode mindestens 20 Jahre Abgeordnete des Europäischen Parlaments waren. Ihr Ausscheiden kann als Akt personeller Erneuerung und Verjüngung der SPD-Abgeordneten verstanden werden. Auch bei der CDU sind vor allem Abgeordnete der Geburtsjahrgänge zwischen 1944 und 1951 nicht mehr nominiert worden, und sie gehörten - mit einer Ausnahme - ebenfalls zwischen 15 und 35 Jahre dem EP an. Auch dieses Ausscheiden aus dem Parlament kann als

11 Von den zwei Abgeordneten, die nicht mehr aufgestellt wurden, wurde eine (Sabine Wils) wegen europapolitischer Differenzen zu den Parteipositionen nicht erneut nominiert und einer (Jürgen Klute) verzichtete wegen der - nach seiner Einschätzung - von der Parteimehrheit vertretenen anti-europäischen Politik auf eine erneute Kandidatur. 


\begin{tabular}{|l|c|c|c|c|c|c|cr|}
\hline \multicolumn{2}{|c|}{ Tabelle 2: Gründe für das Ausscheiden aus dem Europäischen Parlament 2014 (absolut und in } \\
Prozent)
\end{tabular}

Beitrag (wie freiwillig im Einzelnen auch immer) für eine Erneuerung und Verjüngung der EP-Mitglieder der CDU aufgefasst werden. Dass nicht sämtliche Europa-Abgeordneten der CSU erneut unter den ersten acht aufgestellten Kandidaten zu finden waren, sondern nur sieben, resultierte aus dem altersbedingten Verzicht der nachgerückten Gabriele Stauner (geb. 1948) auf eine erneute Nominierung. ${ }^{12}$

Gemessen an den realisierten Mandaten liegt die Kontinuitätsquote bei 79,3 Prozent für alle Parteien; bei Parteien, die Mandatsverluste erlitten (vor allem CSU und FDP, etwas abgeschwächter auch die CDU), war sie erheblich höher.

\section{Ausscheiden aus dem EP mit Ende der Wahlperiode (Verzicht auf Kandidatur, Nicht-Nominierung, Wahlergebnis)}

Von den 99 Abgeordneten aus Deutschland, die 2014 ein Mandat im Europäischen Parlament hatten, kandidierten 27 nicht mehr für die Wahl im Mai 2014, und weitere sieben, die aufgestellt worden waren, scheiterten am Wählervotum (vgl. Tabelle 2). Der größte Teil der Abgeordneten verzichtete freiwillig auf eine erneute Kandidatur (55,9 Prozent). Überwiegend war das erreichte Lebensalter, meist in Verbindung mit einer langen Mitgliedschaft im EP, ausschlaggebend für diesen Schritt (47,1 Prozent). ${ }^{13}$ Drei Abgeordnete der FDP

12 Gabriele Stauner war bereits von 1999 bis 2004 Abgeordnete des EP und erneut als Nachrückerin von 2006 bis 2009. Bei der EP-Wahl 2009 verpasste sie, nominiert auf Platz neun der CSU-Liste, die Erneuerung ihres Mandats nur knapp. Doch kam sie im Oktober 2013 als Nachrückerin wieder ins EP, als Anja Weisgerber ihren Sitz zugunsten eines Bundestagsmandats zurückgab.

13 Von den 27 nicht mehr nominierten Abgeordneten waren 70,3 Prozent 60 Jahre und älter. 
konnten nicht mehr nominiert werden und verzichteten auf eine Kandidatur. ${ }^{14}$ Unfreiwillig verpassten 13 MdEP (38,2 Prozent) ein neuerliches Mandat im EP. Von diesen konnten sieben aufgestellte Abgeordnete aufgrund des Ergebnisses der Wahl ihr Mandat nicht erneuern (20,6 Prozent). Vier scheiterten bereits auf dem Nominierungsparteitag ihrer Partei, und zwei weitere erklärten, aufgrund von politischen Differenzen mit ihrer jeweiligen Partei nicht erneut kandidieren zu wollen. Außerdem konnte eine Abgeordnete der Grünen, die 2013 als Nachrückerin erneut ins EP gelangt war, nicht damit rechnen, ausreichende Unterstützung für eine aussichtsreiche Kandidatur zu erhalten (erfasst unter: unklar / sonstige).

Auch für die Europa-Wahl 2014 ist festzustellen, dass Abgeordnete nur zu einem geringen Teil aufgrund des Wahlergebnisses aus dem Parlament ausschieden. Vorherrschend war ein freiwilliger Verzicht auf eine erneute Kandidatur, und in wenigen Fällen verhinderten parteiinterne Konflikte eine Re-Nominierung und eine Erneuerung des Mandats.

\section{Altersstruktur der EP-Abgeordneten}

Der relativ hohe Anteil re-nominierter EP-Abgeordneter bei den bereits im Europäischen Parlament vertretenen Parteien und ein Wahlergebnis, das ausschließlich (CSU, FDP) oder - mit Ausnahme der SPD - ganz überwiegend (Die Linke, Die Grünen, CDU) zur Wiederwahl von EP-Abgeordnete führte, hat - im Vergleich zur vorangegangenen Wahlperiode - ein moderates Ansteigen des Altersdurchschnitts der Abgeordneten zur Folge. Entsprechend findet sich für die 8. Wahlperiode eine etwas stärkere Vertretung der Altersgruppen ab „50 bis 59 Jahre“ (vgl. Tabelle 3). Der Gruppe „70 bis 79 Jahre“ gehören indes ausschließlich drei Abgeordnete der erstmals im EP vertretenen Parteien an (AfD und ÖPD).

Der Altersschwerpunkt liegt wie in den früheren Wahlperioden bei den 50- bis 59-Jährigen (43,8 Prozent), flankiert durch die beiden angrenzenden Altersgruppen ( 40 bis 49 Jahre: 20,8 Prozent und 60 bis 69 Jahre: 21,9 Prozent). Auch durch den Einzug von acht neuen Parteien mit insgesamt 14 Abgeordneten (14,6 Prozent) hat sich die Altersstruktur der Parlamentsmitglieder nicht auffällig verändert.

Längeres (partei-)politisches Engagement und praktische politische Erfahrungen gelten als Voraussetzungen für eine parteiintern erfolgversprechende Kandidatur und Nominierung für die EP-Wahlen und für eine professionelle Wahrnehmung des Mandats im EP. Diese Anforderungen und Voraussetzungen für eine Kandidatur wirken sich auf die Altersstruktur aller EP-Mitglieder (vgl. Tabelle 3) und auch auf die Altersstruktur der 2014 erstmals ins EP gewählten Abgeordneten aus. Von den 31 Neulingen gehören 41,9 Prozent der Altersgruppe 50 bis 59 Jahre und 25,8 Prozent der Altersgruppe 40 bis 49 Jahre an. Aber auch die Nominierung von jüngeren Abgeordneten (zusammen sechs MdEP) setzt bei den etablierten Parteien ein mehrjähriges parteibezogenes Engagement voraus.

Im Vergleich zu den vorhergehenden Wahlperioden zeigt sich eine „Alterung" der EuropaAbgeordneten durch das Zusammenspiel mehrerer Faktoren: geringe personelle Erneuerung bei den nominierten EP-Kandidaten, lange Verweildauer der Mandatsträger im EP, hohe Anforderungen an parteipolitischem Engagement und an politischer Vorerfahrung als Voraussetzungen für eine Aufstellung sowie eine (zunehmende) Nominierungskonkurrenz. 


\begin{tabular}{|c|c|c|c|c|c|c|c|c|c|c|c|c|}
\hline Tabelle 3: & $\begin{array}{l}\text { Alte } \\
\text { in } P\end{array}$ & rukt & tur der d & & en $M c$ & & 5. $b i$ & & ablpe & de (1999 & s 2014), & solut una \\
\hline & & & \begin{tabular}{|c|} 
Durch- \\
schnitts-
\end{tabular} & & $\begin{array}{l}29 \\
\text { hre }\end{array}$ & $\begin{array}{r}30 \mathrm{~b} \\
\mathrm{Ja}\end{array}$ & $\begin{array}{l}\text { bis } 39 \\
\text { hre }\end{array}$ & $\begin{array}{r}40 \mathrm{l} \\
\mathrm{Ja}\end{array}$ & $\begin{array}{l}\text { bis } 49 \\
\text { ahre }\end{array}$ & $\begin{array}{c}50 \text { bis } 59 \\
\text { Jahre }\end{array}$ & $\begin{array}{c}60 \text { bis } 69 \\
\text { Jahre }\end{array}$ & $\begin{array}{c}70 \text { bis } 79 \\
\text { Jahre }\end{array}$ \\
\hline & $\mathrm{N}$ & $\%$ & alter* & & $\%$ & $\mathrm{~N}$ & $\%$ & $\mathrm{~N}$ & $\%$ & $\mathrm{~N} \quad \%$ & $\mathrm{~N} \quad \%$ & $\mathrm{~N} \quad \%$ \\
\hline $\begin{array}{l}\text { 5. WP } \\
(1999 \text { bis } \\
2004)\end{array}$ & 99 & 100 & 49,0 & 4 & 4,0 & 8 & 8,1 & 36 & 36,4 & $40 \quad 40,4$ & $10 \quad 10,1$ & $1 \quad 1,0$ \\
\hline $\begin{array}{l}\text { 6. WP } \\
(2004 \text { bis } \\
2009)\end{array}$ & 99 & 100 & 50,4 & 4 & 4,0 & 13 & 13,1 & 25 & 25,3 & $40 \quad 40,4$ & $17 \quad 17,2$ & - \\
\hline $\begin{array}{l}\text { 7. WP } \\
\text { (2009 bis } \\
2014)\end{array}$ & 99 & 100 & 51,0 & 3 & 3,0 & 14 & 14,1 & 22 & 22,2 & $39 \quad 39,4$ & $21 \quad 21,2$ & - \\
\hline $\begin{array}{l}\text { 8. WP } \\
\text { (seit 2014) }\end{array}$ & 96 & 100 & 53,3 & 2 & 2,1 & 8 & 8,3 & 20 & 20,8 & $42 \quad 43,8$ & $21 \quad 21,9$ & 33,1 \\
\hline $\begin{array}{l}\text { Anmerkung } \\
* \text { Zu Begir } \\
\text { Datengrun } \\
\text { zung). } \\
\text { Zur Klassif } \\
\text { Wahltag) ir } \\
\text { (Lebensjaht } \\
\text { das Alter m } \\
\text { datum. } \\
\text { Quelle: Eig }\end{array}$ & $\begin{array}{l}\text { dlage } \\
\text { izieru } \\
\text { n Rel } \\
\text { r) zu } \\
\text { ionats }\end{array}$ & $\begin{array}{l}\text { sind d } \\
\text { g: Als } \\
\text { tion zu } \\
\text { festg } \\
\text { fenau } \\
\text { usamm }\end{array}$ & $\begin{array}{l}\text { ie gewähl } \\
\text { Stichtag } \\
\text { um Gebu } \\
\text { elegten St } \\
\text { erhoben, } \\
\text { nenstellun }\end{array}$ & vurd & $\begin{array}{l}\text { d mögl } \\
\text { en. } \\
\text { bgeor } \\
\text { e jewei } \\
\text { um; di } \\
\text { für } \\
\text { cum m }\end{array}$ & $\begin{array}{l}\text { Is de } \\
\text { ie Alt } \\
\text { die } \mathrm{E} \\
\text { it der }\end{array}$ & $\begin{array}{l}\text { r Tag d } \\
\text { tersgru } \\
\text { Errechn } \\
\text { m Tag }\end{array}$ & $\begin{array}{l}\text { r ko } \\
\text { pen } \\
\text { ung } \\
\text { er k }\end{array}$ & $\begin{array}{l}\text { nstitui } \\
\text { erfasse } \\
\text { des Du } \\
\text { onstitui }\end{array}$ & $\begin{array}{l}\text { Wahlperiod } \\
\text { renden Sitz } \\
\text { danach d } \\
\text { chschnittsa } \\
\text { erenden Sit }\end{array}$ & $\begin{array}{l}\text { (konstitui } \\
\text { ng gewähl } \\
\text { jeweilige } \\
\text { ters der M } \\
\text { ang des EP }\end{array}$ & $\begin{array}{l}\text { rende Sit- } \\
\text { (nicht der } \\
\text { ebensalter } \\
\text { EP wurde } \\
\text { ls Bezugs- }\end{array}$ \\
\hline
\end{tabular}

Bemerkenswert für die 8. Wahlperiode ist der hohe Anteil von Frauen in der Altersgruppe 50 bis 59 Jahre (65,7 Prozent). Er liegt etwa 20 Prozentpunkte über den Anteilen der vorangegangenen Wahlperioden und ist mehr als doppelt so groß ist wie bei den männlichen MdEP in dieser Altersgruppe (31,1 Prozent). Die Gründe liegen wiederum in einer Kombination von Mandatskontinuität auch bei den weiblichen MdEP aller Parteien, einer geringen Erneuerung von EP-Mandaten insbesondere bei den Parteien mit relativ hohem Frauenanteil (vor allem Die Grünen, Die Linke) sowie einem relativ hohen Einstiegsalter der Frauen als EP-Abgeordnete (vor allem bei der SPD: fünf der sechs neu ins EP gewählten SPD-Frauen gehörten der Altersgruppe 50 bis 59 Jahre an).

\section{Schul-und Bildungsabschlüsse}

Auch die Abgeordneten der 8. Wahlperiode des EP verfügen über ein hohes Schulbildungsniveau (vgl. Tabelle 4). Über 80 Prozent von ihnen besitzen die Hochschulreife oder eine gleichwertige Qualifikation. Ein Volks- oder Hauptschulabschluss bildet inzwischen eine rar gewordene Ausnahme, während weiterhin eine kleine Gruppe von Abgeordneten die Mittlere Reife (oder einen äquivalenten Schulabschluss) erlangt hat. Diese Gruppe ist seit der 4. Wahlperiode etwa gleich groß geblieben.

Eine Aufschlüsselung der Schul- und Bildungsabschlüsse nach Parteien ist angesichts der geringen Anzahl der Abgeordneten bei den einzelnen Parteien nur sehr begrenzt für Interpretationen zugänglich. 


\begin{tabular}{|c|c|c|c|c|c|c|c|c|}
\hline & $(1995$ & $\begin{array}{l}\text { XP } \\
\text { s 2004) }\end{array}$ & $(200$ & $\begin{array}{l}\text { VP } \\
s \text { 2009) }\end{array}$ & $(200$ & $\begin{array}{l}\text { VP } \\
\text { s 2014) }\end{array}$ & & $\begin{array}{l}\text { XP } \\
2014)\end{array}$ \\
\hline & $\mathrm{N}$ & $\%$ & $\mathrm{~N}$ & $\%$ & $\mathrm{~N}$ & $\%$ & $\mathrm{~N}$ & $\%$ \\
\hline Volksschule / Hauptschule & 2 & 2,0 & 2 & 2,0 & 1 & 1,0 & 1 & 1,0 \\
\hline Mittlere Reife & 11 & 11,1 & 4 & 8,1 & 9 & 9,1 & 12 & 12,5 \\
\hline $\begin{array}{l}\text { Abitur / Fachhochschul-/ } \\
\text { Hochschulreife }\end{array}$ & 81 & 81,8 & 84 & 84,8 & 82 & 82,8 & 79 & 82,3 \\
\hline sonstige / unklar & 5 & 5,1 & 5 & 5,1 & 7 & 7,1 & 4 & 4,2 \\
\hline insgesamt & 99 & 100 & 99 & 100 & 99 & 100 & 96 & 100 \\
\hline $\begin{array}{l}\text { Studium } \\
\text { (Lehrerseminar, Fachhoch- } \\
\text { schule, Hochschule, Univer- } \\
\text { sität, Akademie der Arbeit) }\end{array}$ & 83 & 83,8 & 84 & 84,8 & 79 & 79,8 & 78 & 81,3 \\
\hline
\end{tabular}

Als inzwischen typisch für Europa-Abgeordnete ${ }^{15}$ gilt ein qualifiziert abgeschlossenes Studium, das seit der 2. Wahlperiode des EP etwa 80 Prozent der Abgeordneten vorweisen können. Nicht ein spezifisches Studium (zum Beispiel Rechtswissenschaft), sondern „Transferqualifikationen“, die über die Schul- und Weiterbildung sowie durch ein Studium erworben werden können, eröffnen und erleichtern den Zugang zum Berufsfeld „Politik“ und den Weg zur anspruchsvollen Position eines / einer Abgeordneten.

\section{Geschlechterzusammensetzung}

Der Anteil von Frauen an den Abgeordneten hat sich auch mit der 8. Wahlperiode nicht markant verändert: Mit 35 MdEP stellen sie einen Anteil von 36,5 Prozent (vgl. Tabelle 5).

Überdurchschnittlich ist weiterhin der Anteil von Frauen bei den SPD-Abgeordneten, während sich ihr Anteil bei der CDU ohne auffällige Veränderungen noch immer auf einem recht niedrigen Niveau (17,2 Prozent) bewegt. Für die geringe Zahl weiblicher Abgeordneter bei der CDU ist in erster Linie das Nominierungsverfahren über Landeslisten ausschlaggebend. ${ }^{16}$ Die SPD, die einen Frauenanteil von 48,1 Prozent aufweist, stellt über

15 Inzwischen auch ähnlich für die Parlamentarier des Bundestags und der Landesparlamente, vgl. Werner Reutter, Föderalismus, Parlamentarismus und Demokratie. Landesparlamente im Bundesstaat, Opladen 2008, S. 132 ff.; Michael F. Feldkamp, Datenhandbuch zur Geschichte des Deutschen Bundestages 1990 bis 2010, Baden-Baden 2011, S. 299 - 303.

16 Da bei der CDU die Bundespartei keine faktisch wirksamen Vorgaben für den Anteil von Frauen an den Parlamentsmitgliedern macht ( $\$ 15$ des Statuts der CDU bietet bestenfalls Orientierung) und folglich auch keine Regulierung in Form einer geschlechteralternierenden Kandidatenliste kennt, hängt der Frauenanteil von der Gestaltung der Landeslisten ab; diese sind jedoch durchgehend in der Spitzenposition und meist auch auf den folgenden Listenplätzen männlich besetzt, so dass nur wenige Frauen überhaupt eine Chance haben, über eine Landesliste ins EP zu gelangen. Bei der EP-Wahl 2014 gelang das nur Birgit Collin-Lange: Platz zwei (von zwei erfolgreichen Li- 


\begin{tabular}{|c|c|c|c|c|c|c|c|c|c|c|}
\hline \multirow[t]{3}{*}{ Tabelle 5: } & \multirow{3}{*}{$\mathrm{CDU}$} & \multirow{3}{*}{ CSU } & \multirow{3}{*}{ SPD } & \multirow{3}{*}{ FDP } & \multirow{3}{*}{$\begin{array}{c}\text { Die } \\
\text { Grünen }\end{array}$} & \multirow{3}{*}{$\begin{array}{l}\text { PDS / } \\
\text { Linke }\end{array}$} & \multirow{3}{*}{ AfD } & \multirow{3}{*}{\begin{tabular}{|c|} 
andere \\
Parteien
\end{tabular}} & \multirow{2}{*}{\multicolumn{2}{|c|}{ Insgesamt }} \\
\hline & & & & & & & & & & \\
\hline & & & & & & & & & $\mathrm{N}$ & $\%$ \\
\hline $\begin{array}{l}\text { 5. WP (1999 bis } \\
2004)\end{array}$ & 43 & 10 & 33 & - & 7 & 6 & - & - & 99 & 100 \\
\hline Männer & 31 & 6 & 19 & - & 3 & 3 & - & - & 62 & 62,6 \\
\hline Frauen & 12 & 4 & 14 & - & 4 & 3 & - & - & 37 & 37,3 \\
\hline in Prozent* & 27,9 & 40,0 & 42,4 & - & 57,1 & 50,0 & - & - & & \\
\hline $\begin{array}{l}\text { 6. WP (2004 bis } \\
\text { 2009) }\end{array}$ & 40 & 9 & 23 & 7 & 13 & 7 & - & - & 99 & 100 \\
\hline Männer & 32 & 7 & 14 & 6 & 6 & 3 & - & - & 68 & 68,7 \\
\hline Frauen & 8 & 2 & 9 & 1 & 7 & 4 & - & - & 31 & 31,3 \\
\hline in Prozent* & 20,0 & 22,2 & 39,1 & 14,3 & 53,8 & 57,1 & - & - & & \\
\hline $\begin{array}{l}\text { 7. WP (2009 bis } \\
\text { 2014) }\end{array}$ & 34 & 8 & 23 & 12 & 14 & 8 & - & - & 99 & 100 \\
\hline Männer & 26 & 5 & 13 & 7 & 7 & 4 & - & - & 62 & 62,6 \\
\hline Frauen & 8 & 3 & 10 & 5 & 7 & 4 & - & - & 37 & 37,3 \\
\hline in Prozent* & 23,5 & 37,5 & 43,5 & 41,7 & 50,0 & 50,0 & - & - & & \\
\hline 8. WP (seit 2014) & 29 & 5 & 27 & 3 & 11 & 7 & 7 & 7 & 96 & 100 \\
\hline Männer & 24 & 3 & 14 & 2 & 5 & 3 & 5 & 5 & 61 & 63,5 \\
\hline Frauen & 5 & 2 & 13 & 1 & 6 & 4 & 2 & 2 & 35 & 36,5 \\
\hline in Prozent* & 17,2 & 40,0 & 48,1 & 33,3 & 54,5 & 57,1 & 28,6 & 28,6 & & \\
\hline \multicolumn{11}{|c|}{$\begin{array}{l}\text { Anmerkung: Rundungsdifferenzen sind möglich. } \\
* \text { Anteil der Frauen in Prozent aller Abgeordneten der jeweiligen Partei. } \\
\text { Quelle: Eigene Zusammenstellung. }\end{array}$} \\
\hline
\end{tabular}

eine Bundesliste auf, die geschlechteralternierend besetzt wird und mit einem männlichen Kandidaten (2014: Martin Schulz) auf dem ersten Listenplatz beginnt. Der hohe - und bei ungerader Anzahl der Abgeordneten überproportionale - Frauenanteil bei den Parteien Die Linke und Die Grünen resultiert ebenfalls aus einer geschlechteralternierende Nominierung und der Besetzung des ersten Listenplatzes mit einer Frau. Der etwas überraschend hohe - aber keineswegs ungewöhnliche - Frauenanteil bei der CSU (40 Prozent) ist die Folge einer Listenaufstellung mit zwei Frauen auf den ersten fünf Plätzen (Platz 3 und 4).

Der noch immer relativ geringe Frauenanteil im EP resultiert vor allem aus der männlich dominierten Nominierungspraxis der CDU. Verstärkt wird dieser Effekt in der 8. Wahlperiode durch die neu ins EP gelangten Parteien, die - zusammen - eine Frauenquote von nur 28,6 Prozent aufweisen.

stenplätzen) auf der Landesliste von Rheinland-Pfalz; Ingeborg Gräßle: Platz fünf (von fünf) auf der Landesliste von Baden-Württemberg; Godelieve Quisthoudt-Rowohl: Platz drei (von vier) auf der Landesliste von Niedersachsen; Renate Sommer: Platz drei und Sabine Verheyen: Platz fünf (von acht) auf der Landesliste von NRW. 


\section{Parlamentserfahrung der EP-Abgeordneten}

Die Veränderung des im Europa-Parlament vertretenen Parteienspektrums durch den Wegfall der Sperrklausel und durch den relativ hohen Wahlerfolg der AfD hat die parlamentarische Vorerfahrung von EP-Abgeordneten dennoch nicht auffällig vermindert. Die MdEP bringen ein breites parlamentarisches Erfahrungswissen in das Parlament ein (vgl. Tabelle 6). ${ }^{17}$ Dieses speist sich in geringem Umfang aus Abgeordnetentätigkeit im Bundestag (7,3 Prozent) und in etwas größerem Maße aus in Landesparlamenten gewonnenen Erfahrungen (27,1 Prozent) sowie aus der Abgeordnetentätigkeit in vorangegangenen Wahlperioden des EP (68,7 Prozent). Da die Mehrzahl der Abgeordneten zwei und mehr Wahlperioden dem Europäischen Parlament angehören, kumuliert sich diese parlamentarische Vorerfahrung: In der 8. Wahlperiode verfügen 75 Prozent der MdEP über parlamentarische Vorerfahrung (ohne Doppelzählungen). Mit dieser Quote unterscheidet sich die 8. Wahlperiode nicht von den vorangegangenen. Mit einer Ausnahme - Ulrike Müller, die über die Liste Freie Wähler (FW) ein Mandat erlangte und bereits von 2008 bis 2014 als FW-Mitglied dem Bayerischen Landtag angehörte - besitzt keiner der 14 Abgeordneten der erstmals im EP vertretenen Parteien Parlamentserfahrung.

Das Abgeordnetenmandat im EP stellt für viele Mitglieder den politisch-parlamentarischen Schwer- und Höhepunkt ihrer (berufs-)politischen Karriere dar. Diese Feststellung wird nicht relativiert, wenn berücksichtigt wird, dass in der 8. WP sieben Abgeordnete vorher parlamentarische Erfahrungen im Bundestag gesammelt hatten und 26 bereits Mitglied eines Landesparlaments gewesen waren. Zu berücksichtigen ist, dass diese Daten über mehrere Wahlperioden des EP kumulierte parlamentarische Erfahrungen angeben. Von den Abgeordneten, die erstmals in der 8. Wahlperiode Mitglied des EP wurden, hatte nur eine vorher ein Bundestagsmandat und fünf ein Landtagsmandat inne, zusammen 6,2 Prozent der 96 deutschen EP-Abgeordneten beziehungsweise 19,4 Prozent der Neulinge. ${ }^{18}$ Dennoch ist eine Zirkulation zwischen Landes-, Bundes- und Europa-Parlament in keine der beiden Richtungen bislang prägend geworden für die Rekrutierung von Abgeordneten oder für einen inter-parlamentarischen Erfahrungstransfer im europäischen Mehrebenensystem. Denn in den meisten Fällen ging dem Wechsel in das EP ein wahl- oder nominierungsbedingter Mandatsverlust und/oder eine (unfreiwillige) Beendigung einer Regierungsfunktion voraus.

17 Die Parlamentsmandate der MdEP weisen nur einen Teil der politischen Erfahrungen aus; voraus ging bei den meistens Abgeordneten ein Engagement in kommunalen Selbstverwaltungsorganen und innerhalb der Parteigremien, vgl. Karlheinz Reif / Hermann Schmitt / Klaus Schubert, Wer sind und was wollen die Deutschen im Europäischen Parlament? Sozialprofil, politischer Rückhalt und Zielvorstellungen der deutschen Kandidaten zum Europäischen Parlament, in: ZParl, 10. Jg. (1979), H. 3, S. 332 - 354; Rudolf Hrbek / Carl-Christoph Schweitzer, Die deutschen Europa-Parlamentarier. Ergebnisse einer Befragung der deutschen Mitglieder des Europäischen Parlaments, in: APuZ, 39. Jg. (1989), B. 3, S. 3 - 18; Benjamin Höhne, a.a.O. (Fn. 3), S. $226-238$.

18 Die MdEP waren mindestens eine Wahlperiode Mitglied des Bundestages (Iris Hoffmann, SPD: 1998 bis 2009) oder eines Landesparlaments (David McAllister, CDU: 1998 bis 2014; Ulrike Müller, FW: 2008 bis 2014; Susanne Melior, SPD: 2004 bis 2014; Maria Noichl, SPD: 2008 bis 2013; Joachim Schuster, SPD: 1999 bis 2006). 


\begin{tabular}{|c|c|c|c|c|c|c|c|c|c|}
\hline \multicolumn{10}{|c|}{$\begin{array}{l}\text { Tabelle 6: Parlamentarische Erfahrungen der MdEP vor dem Mandat im EP zu Beginn der } \\
\text { 8. Wahlperiode } 2014 \text { (insgesamt und nach Parteien*, absolut und in Prozent) }\end{array}$} \\
\hline & \multicolumn{2}{|c|}{$\begin{array}{c}\text { 8. WP } \\
\text { (seit 2014) }\end{array}$} & \multirow{2}{*}{$\begin{array}{l}\mathrm{CDU} \\
\mathrm{N}\end{array}$} & \multirow{2}{*}{$\begin{array}{l}\mathrm{CSU} \\
\mathrm{N}\end{array}$} & \multirow{2}{*}{$\begin{array}{l}\text { SPD } \\
\mathrm{N}\end{array}$} & \multirow{2}{*}{$\begin{array}{c}\text { Die } \\
\text { Grünen } \\
\mathrm{N}\end{array}$} & \multirow{2}{*}{$\begin{array}{l}\text { FDP } \\
\mathrm{N}\end{array}$} & \multirow{2}{*}{$\begin{array}{l}\text { Die } \\
\text { Linke } \\
\mathrm{N}\end{array}$} & \multirow{2}{*}{$\begin{array}{c}\text { Freie } \\
\text { Wähle } \\
\mathrm{N}\end{array}$} \\
\hline & $\mathrm{N}$ & Prozent & & & & & & & \\
\hline $\mathrm{MdB}^{\mathrm{a}} / \mathrm{VK}$ & 7 & 7,3 & 3 & 1 & 3 & - & - & - & - \\
\hline $\mathrm{MdL}^{\mathrm{b}}$ & 26 & 27,1 & 8 & 2 & 6 & 4 & 2 & 3 & 1 \\
\hline davon nur $\mathrm{MdL}^{\mathrm{c}}$ & 24 & 25,0 & 6 & 2 & 6 & 4 & 2 & 3 & 1 \\
\hline$M d B$ und $M d L^{d}$ & 31 & 32,3 & 9 & 3 & 9 & 4 & 2 & 3 & 1 \\
\hline $\begin{array}{l}\text { MdB / erstes } \\
\text { EP-Mandat in der } \\
\text { 8. WP }\end{array}$ & 1 & 1,0 & - & - & 1 & - & - & - & - \\
\hline $\begin{array}{l}\text { MdLe / erstes } \\
\text { EP-Mandat in der } \\
\text { 8. WP }\end{array}$ & 5 & 5,2 & 1 & - & 3 & - & - & - & 1 \\
\hline $\begin{array}{l}\text { MdEP in vorange- } \\
\text { gangener }(7 . \mathrm{WP})\end{array}$ & 65 & 67,7 & 25 & 5 & 17 & 9 & 3 & 6 & - \\
\hline $\begin{array}{l}\text { MdEP in früherer } \\
\text { WP (diskontinuier- } \\
\text { lich) }\end{array}$ & $1^{\mathrm{f}}$ & 1,0 & - & - & 1 & - & - & - & - \\
\hline $\begin{array}{l}\text { Parlamentserfah- } \\
\text { rung vor der } 8 \text {. WP }\end{array}$ & 72 & 75,0 & 26 & - & 22 & 9 & 3 & 6 & 1 \\
\hline MdEP insgesamt & 96 & 100 & 29 & 5 & 27 & 11 & 3 & 7 & 1 \\
\hline $\begin{array}{l}\text { Parlamentserfah- } \\
\text { rung der jeweiligen } \\
\text { MdEP (in Prozent) }\end{array}$ & - & - & 89,7 & 100 & 81,4 & 81,8 & 100 & 100 & 100 \\
\hline \multicolumn{10}{|c|}{$\begin{array}{l}\text { Anmerkung: Rundungsdifferenzen sind möglich. } \\
\text { * Mit Ausnahme der Freien Wähler verfügte keine der Parteien, die erstmals ins Europäische Parlament } \\
\text { gelangten, über Abgeordnete mit Parlamentserfahrungen auf Landes-, Bundes- oder europäischer Ebene. } \\
\text { a Erfasst werden auch drei Abgeordnete, die } 1990 \text { für wenige Monate Mitglied der freigewählten Volks- } \\
\text { kammer (10. Wahlperiode, 18. März bis 3. Oktober 1990) und des Bundestags (Oktober bis Dezem- } \\
\text { ber 1990) waren. } \\
\text { b Mit der Abkürzung MdL (= Mitglied des Landtags) werden auch die Mitglieder des Berliner Abgeord- } \\
\text { netenhauses, der Bremer und der Hamburger Bürgerschaft erfasst. } \\
\text { c Die in dieser Zeile ausgewiesenen Abgeordneten waren ausschließlich Mitglieder eines Landesparla- } \\
\text { dents. } \\
\text { e Ohne Doppelzählungen. } \\
\text { f Ohne MdL, die auch Mitglied des Bundestages waren. } \\
\text { Quelle: Eigene Zusammenstellung. }\end{array}$} \\
\hline
\end{tabular}




\section{Regierungserfahrung}

Nur wenige frühere Kabinettsmitglieder übernehmen nach Beendigung ihrer Regierungsfunktion ${ }^{19}$ ein Mandat im Europäischen Parlament. ${ }^{20}$ Der Wechsel von einem Amt (Minister, Staatssekretär) in der Bundesregierung ins EP kann als Ausnahme angesehen werden. Die Wahrnehmung einer Funktion in der Bundesregierung und die damit gewonnenen Erfahrungen „berechtigen“ offensichtlich zu anderen Aufgaben. Ebenso selten ist die Fortsetzung einer politischen Karriere nach Beendigung des Amtes des Ministerpräsidenten durch ein Mandat im Europäischen Parlament. Es ist nicht abzuschätzen, ob und mit welchem Ziel sich David McAllister ${ }^{21}$ langfristig europapolitisch engagieren wird. Nach der verlorenen Landtagswahl in Niedersachsen (2013) suchte er eine neue politische Aufgabe außerhalb der Landespolitik und konnte als (wenig wahrgenommener) Spitzenkandidat der CDU 2014 ins EP einziehen.

Auch die Übernahme eines EP-Mandats durch ehemalige Minister oder Staatssekretäre in Landesregierungen erfolgte erst nach dem Verlust des Regierungsamtes. Auch dieser Wechsel von der exekutiven Funktion (meistens verbunden mit einem Mandat im Landesparlament) zu einer legislativen und kontrollierenden Aufgabe im Europäischen Parlament kommt selten vor. Mit Beginn der 8. Wahlperiode setzte nur ein früheres Mitglied einer Landesregierung (Joachim Schuster, SPD, Staatsrat im Bremer Senat 2006 bis 2012) seine politische Karriere als Abgeordneter im EP fort. ${ }^{22}$ Außer ihm gehören dem Europäischen Parlament gegenwärtig noch fünf ehemalige Minister und Staatssekretäre aus Landesregierungen an, die bereits zwischen einer und vier Wahlperioden Mitglied des EP sind. ${ }^{23}$ An dem seit der 2. Wahlperiode festzustellenden geringen Kompetenztransfer von einer Position in einer Landes- oder Bundesregierung in das Europa-Parlament hat sich - erwartungsgemäß - auch in der 8 . Wahlperiode nichts geändert.

\section{Dauer der Parlamentsmitgliedschaft}

Wie bei allen demokratischen Parlamenten kommen auch beim EP zwei konkurrierende Funktionsanforderungen zur Geltung: zum einen die demokratietheoretisch begründete

19 Die Übernahme einer Kandidatur für ein EP-Mandat erfolgt nach einem (meist) wahlbedingten Verlust des ausgeübten Regierungsamtes. Ein selbstgewählter Wechsel von einer (noch ausgeübten) Regierungsfunktion auf Landes- oder Bundesebene richtet sich nicht auf ein Mandat im Europäischen Parlament, sondern auf eine Funktion in der einflussreicheren administrativen/ exekutiven Institution der EU, in der Kommission.

20 Vgl. Peter Rütters, Daten zur Sozialstruktur der deutschen Abgeordneten, a.a.O. (Fn. 8), S. 777, Tabelle 9.

21 David McAllister (CDU), Ministerpräsident in Niedersachsen 2010 bis 2013, MdEP seit 2014.

22 In der vorangegangenen Wahlperiode (2009 bis 2014) waren es drei ehemalige Landesminister / Staatssekretäre, in der 6. WP (2004 bis 2009) war es eine, und in der 5. WP waren es wiederum drei, die ein EP-Mandat übernahmen.

23 Landesminister: Werner-Josef Langen (CDU), Landesminister Rheinland-Pfalz 1990 bis 1991, MdEP seit 1994; Jo Leinen (SPD), Landesminister im Saarland, 1985 bis 1994, MdEP seit 1999; Helga Türpel (Grüne), Senatorin für Kultur im Bremer Senat 1991 bis 1995, MdEP seit 2004; Monika Hohlmeier (CSU), Staatssekretärin (1993 bis 1998), Landesministerin (1998 bis 2005) in Bayern, MdEP seit 2009; Hermann Winkler (CDU), Staatsminister in Sachsen-Anhalt 2004 bis 2007, MdEP seit 2009; Joachim Schuster (SPD), Staatsrat in Bremen, 2006 bis 2012, MdEP seit 2014. 
Anforderung an jeden Abgeordneten, jeweils nur für eine begrenzte Zeit (meist: Wahl- I Legislaturperiode) durch allgemeine Wahlen mit der Aufgabe der parlamentarischen Repräsentation betraut zu werden und gegebenenfalls durch erneute Wahl die Legitimation für ein weiteres zeitlich begrenztes Parlamentsmandat zu erhalten (Wiederwahl); zum anderen die institutionelle Anforderung, durch die Erfahrungen einer längeren Abgeordnetentätigkeit die komplexen Funktionsanforderungen eines Parlaments zu bewältigen. Hinzu kommt das Interesse der Abgeordneten an berufspolitischer und sozialer Absicherung durch eine lange Parlamentsmitgliedschaft. Noch einmal sei hervorgehoben: die Mehrzahl der EP-Abgeordneten übt das europa-politische und europa-parlamentarische Engagement weder beiläufig noch sporadisch aus.

Veränderungen der Mitgliederstruktur des EP wurden - wie erwähnt - bei der Wahl 2014 durch vier Faktoren beeinflusst: (1) durch eine geringe Reduktion der Gesamtzahl der Abgeordneten von 99 auf 96; (2) durch die Zunahme der im EP vertretenen Parteien als Folge der Aufhebung der Speerklausel; (3) durch Veränderungen der Anzahl der Mandate, die die einzelnen Parteien aufgrund ihres Wahlergebnisses erlangen konnten; (4) durch parteiinterne Entscheidungen über die Aufstellung der Listen für die Europawahl.

Während die Reduktion der Gesamtzahl der Mandate nur marginale Auswirkungen hat, führten der Wegfall der Sperrklausel und der Wahlerfolg der AfD dazu, dass die Abgeordneten der erstmals im EP vertretenen Parteien zusammen 14,6 Prozent aller MdEP (absolut: 14) ausmachen und einen Anteil von 45,2 Prozent der insgesamt 31 neu ins EP gelangten deutschen Parlamentarier/innen stellen.

Der Anteil der neu ins EP gewählten Abgeordneten liegt mit 32,3 Prozent aller MdEP im langfristigen Trend des Personalwechsels seit der zweiten Wahlperiode trotz der acht erstmals im Europa-Parlament vertretenen Parteien (vgl. Tabelle 7). Komplementär verhält sich die Kontinuität der Mitgliedschaft (insgesamt 67,7 Prozent). Ein Drittel der MdEP war bereits in der vorangegangenen 7. Wahlperiode (2009 bis 2014) gewählt worden, und ein weiteres Drittel gehörte dem EP zwei und mehr Wahlperioden an. Nach wie vor kennzeichnet das EP - nach diesen Daten - ein ausgeprägtes Interesse der Parteien und der Abgeordneten an einem langfristigen Engagement, die Aufrechterhaltung und Bewahrung europapolitischer Kompetenzen sowie eine meist moderate personelle Erneuerung.

Erhebliche Abweichungen von den Durchschnittswerten zeigen sich bei einzelnen Parteien. Dies wird besonders deutlich im Vergleich von SPD und CDU/CSU. Die Unionsparteien weisen zusammen, bedingt durch Wahl- und Mandatsverluste (von 42 auf 34 MdEP) und einen zurückhaltenden Kandidatenwechsel, eine sehr hohe AbgeordnetenKontinuität von 88,2 Prozent auf (38,2 Prozent gehören mehr als zwei Wahlperioden dem EP an). ${ }^{24}$ Hingegen lag die Kontinuitätsquote der SPD-Abgeordneten nur bei 63 Prozent. Der überdurchschnittliche Anteil von neuen MdEP resultierte aus dem Wahl- und Mandatsgewinn der Partei (von 23 auf 27 Mandate) und aus dem Verzicht älterer und langjähriger Abgeordneter auf eine erneute Nominierung, so dass insgesamt zehn Abgeordnete (37 Prozent) erstmals über die SPD-Liste ins EP einziehen konnten. Da bis auf die SPD alle etablierten Parteien zurückhaltend bei der Aufstellung neuer Kandidaten waren und bei der EP-Wahl 2014 Mandate einbüßten, hat dies eine sehr hohe Abgeordneten-Kontinuität zur

24 Aufgrund der Stimmenverluste bei den Wahlen musste die CSU, die keine neuen Abgeordneten ins EP entsenden konnte, sogar auf erfahrene Parlamentarier wie Bernd Posselt verzichten, dem auf dem sicher geglaubten 6. Listenplatz nicht mehr der Einzug ins EP gelang. 


\section{Europa-Wahl 2014: kaum Veränderungen des Sozialprofils der deutschen MdEP}

Zwei Faktoren bestimmten vor allem die Europa-Wahl im Jahr 2014: der Wegfall der Sperrklausel von fünf Prozent, was den Zugang zum Europäischen Parlament auch Parteien eröffnete, die eine sehr geringe Wählerschaft für sich zu mobilisieren vermochten, und die erfolgreiche Wahlbeteiligung von europa-skeptischen und rechtspopulistischen Parteien wie der AfD. Während der Einzug von sieben Ein-Mandat-Parteien ins EP als zweifelhafter Gewinn für die demokratische Repräsentation der Bürger angesehen werden kann, sind die dadurch bewirkten Veränderungen des Sozialprofils der deutschen Europa-Abgeordneten dennoch gering.

Die 14 Abgeordneten der neu im EP vertretenen Parteien weisen nur geringe Variationen zum bisherigen Sozialprofil der MdEP auf. Weder unterscheidet sich ihre Altersstruktur noch ihr Schul- und Bildungsniveau in relevanter Weise von den Parlamentsmitgliedern der etablierten Parteien. ${ }^{25}$ Der geringe Anteil von Frauen unter den Abgeordneten sowohl bei der AfD (28,6 Prozent) als auch bei den sieben Ein-Mandat-Parteien, von denen nur zwei eine Frau auf dem erfolgreichen ersten Listenplatz nominiert hatten (Freie Wähler, Piraten), hebt sich von den etablierten Parteien - mit Ausnahme der CDU - ab. Die Folgen für die Geschlechterrelation der EP-Abgeordneten insgesamt sind jedoch marginal. Die nur geringen Abweichungen zum durchschnittlichen Sozialprofil sind vor allem darauf zurückzuführen, dass auch für die „neuen“, bisher überwiegend außerparlamentarisch aufgetretenen Parteien ähnliche Rekrutierungsbedingungen für Spitzenpolitiker und Kandidaten für Parlamentspositionen gelten wie bei den etablierten Parteien.

Veränderungen im Sozialprofil erfolgten überwiegend durch indirekte Auswirkungen des Einzugs der Abgeordneten der „neuen“ Parteien in das EP. Dass - bis auf die SPD - alle etablierten Parteien Mandatsverluste hinnehmen mussten, hat die Möglichkeit für eine personelle Erneuerung vermindert oder gänzlich verhindert. Bei der CDU führte dies auch zu einem Absinken der im Vergleich zu den anderen Parteien bereits niedrigen Frauenquote. Die hohe Re-Nominierungsquote bewirkt eine leichte „Alterung“ der EP-Mitglieder der Parteien, die Mandatsverluste hinnehmen mussten. Aber auch die SPD hatte an diesem Trend teil, jedoch als Folge des hohen Anteils der Altersgruppe 50 bis 59 Jahre (70 Prozent) bei den neu ins EP entsandten Abgeordneten.

Die Folgen der Veränderung im Wahlrecht für die Europa-Wahl 2014 und im Abstimmungsverhalten der Wahlbürger zeigen sich kaum in relevanten Veränderungen der Sozialstruktur der EP-Abgeordneten. Die Auswirkungen werden vor allem im politischen Bereich und im parlamentarischen Handeln der Abgeordneten zu finden sein. Die Vertreter der Ein-Mandat-Parteien werden kaum in der Lage sein, die Repräsentationsanforderungen eines parlamentarischen Mandats zu erfüllen und konstruktiv im Europäischen Parlament mitzuwirken.

25 Dass drei Abgeordnete der Altersgruppe 70 bis 79 Jahre ein Mandat für das EP erlangten (HansOlaf Henkel und Joachim Starbatty für die AfD; Klaus Buchner für die ÖDP), verweist vor allem auf eine zeitlich begrenzte politische, parlamentarische und berufspolitische Perspektive dieser Parlamentarier. 